unreliable for the estimation of GFR. The patients most often collected the 24 hour urine on an outpatient basis, and we believe that these unsatisfactory results can be caused by imprecise urine collection.

It is evident from the present study and the above mentioned reports that the combination regimens of platinum and podophyllin derivatives can produce high response rates in the treatment of extensive SCLC, but it is rather disappointing that response duration and survival time remain very short. A possible step forward could be dose intensification combined with haematopoietic growth factors.

1. Evans WK, Shepherd FA, Feld R, Osaba D, Dang P, Deboer G. VP-16 and cisplatin as first-line therapy for small-cell lung cancer. f Clin Oncol 1985, 3, 1471-1477.

2. Sierocki JS, Hilaris BS, Hopfan S, et al. Cis-dichlorodiammineplatinum (II) and VP-16-213: An active induction regimen for small cell carcinoma of the lung. Cancer Treat Rep 1979, 63, 1593-1597.

3. Smith IE, Harland SJ, Robinson BA, et al. Carboplatin: a very active new cisplatin analog in the treatment of small cell lung cancer. Cancer Treat Rep 1985, 69, 43-46.

4. Bork E, Hansen M, Dombernowsky P, Hansen SW, Pedersen AG, Hansen HH. Teniposide (VM-26), an overlooked highly active agent in small-cell lung cancer. Results of a phase II trial in untreated patients. 7 Clin Oncol 1986, 4, 524-527.

5. Hansen $\mathrm{HH}$. Staging of small cell anaplastic carcinoma of lung. In: Williams CJ and Whitehouse JMA, eds. Recent Advances in Clinical Oncology. Edinburgh, Churchill Livingstone, 1980, 285-294.
6. WHO. Handbook in Reporting Results on Cancer Treatment. WHO Offset Publication No. 48, Geneva, World Health Organization, 1979.

7. Smith IE, Evans BD, Gore ME, et al. Carboplatin (paraplatin; JM8) and etoposide (VP-16) as first-line combination therapy for small-cell lung cancer. F Clin Oncol 1987, 5, 185-189.

8. Bishop JF, Raghavan D, Stuart-Harris R, et al. Carboplatin (CBDCA, JM-8) and VP-16-213 in previously untreated patients with small-cell lung cancer. $\mathcal{F}$ Clin Oncol 1987, 5, 1574-1578.

9. Evans WK, Eiscnhauer E, Hughes P, et al. VP-16 and Carboplatin in previously untreated patients with extensive small cell lung cancer: a study of the National Cancer Institute of Canada Clinical Trials Group. Br f Cancer 1988, 58, 464-468.

10. Goss G, Vincent $M$, Bozek T, et al. A phase II study of teniposide (VM 26) and carboplatinum (Cp) in patients with limited and extensive small cell lung cancer (SCLC). Proc Am Soc Clin Oncol $1989,8,222$.

11. Calvert AH, Harland SJ, Newell DR, et al. Early clinical studies with cis-diammine-1, 1-cyclobutane dicarboxylate platinum II. Cancer Chemother Pharmacol 1982, 9, 140-147.

12. Egorin MJ, Van Echo DA, Tipping SJ, et al. Pharmacokinctics and dosage reduction of cis-diammine(1,1-cyclobutanedicarboxylato)platinum in patients with impaired renal function. Cancer Res 1984, 44, 5432-5438.

13. Calvert AH, Newell DR, Gumbrell LA, et al. Carboplatin dosage: Prospective evaluation of a simple formula based on renal function. f Clin Oncol 1989, 7, 1748-1756.

Acknowledgements-Dr $\Lambda$ rnc Scll is acknowledged for assisting the response evaluation by reviewing X-ray pictures. Expertise on use of the database was kindly provided by $2:$ Peter Vejby Hansen, the Danish Cancer Society.

\title{
Ifosfamide in Advanced Adenocarcinoma of the Oesophagus or Oesophageal-Gastric Junction Area
}

\author{
Tjebbe C. Kok, Ate van der Gaast, Ted A.W. Splinter and Huug W. Tilanus \\ for the Rotterdam Esophageal Tumor Study Group
}

25 previously untreated patients with inoperable or metastatic adenocarcinoma of the oesophagus or oesophageal-gastric junction area were treated with ifosfamide $6 \mathrm{~g} / \mathrm{m}^{2}$ over 48 hours, combined with mesna $6 \mathrm{~g} / \mathrm{m}^{2}$. 1 complete response and 1 partial response were seen among 23 patients evaluable, with a response duration of $29+$ months and 7 months, respectively. Toxicity was not severe: grade 3 infection in 2 patients, grade 3 leucopenia in 3 patients and grade 3 nausea in 4 patients. No life-threatening episodes or central nervous system toxicity were encountered. Ifosfamide has limited activity in adenocarcinoma of the oesophageal-gastric junction area.

Eur 7 Cancer, Vol. 27, No. 9, pp. 1112-1114, 1991.

\section{INTRODUCTION}

THE OUTLOOK for patients with adenocarcinoma of the oesophagus is dismal; in about $40 \%$ metastatic disease is apparent at first presentation. Even if a patient is operable, the 5-year survival after surgery with curative intent is $<10 \%$. Most of these patients die with distant metastases. Obviously, there is a need for effective chemotherapy. We investigated the activity and toxicity of ifosfamide.

\section{PATIENTS AND METHODS}

Until July 1990, 25 consecutive previously untreated patients were entered in the study. The main eligibility criteria were histologically proven adenocarcinoma of the oesophagus or oesophageal-gastric junction area, with or without Barrett's epithelium (patients with adenocarcinoma of the gastric cardia, without involvement of the oesophageal-gastric junction area were not eligible); performance status (Karnofsky) $>60 \%$ and 
Table 1. Characteristics of 23 evaluable patients

\begin{tabular}{lc}
\hline Male/female & $21 / 2$ \\
Median age (yr) & $55(30-74)$ \\
Median performance (Karnofsky) & $80(60-100) \%$ \\
Extent of disease & \\
Locally advanced & 2 \\
Primary excised, metastases & 4 \\
$\quad$ Intra-abdominal & 1 \\
Liver & 1 \\
Pleural & 1 \\
Skin & 1 \\
Primary plus metastases & 17 \\
Intra-abdominal & 2 \\
Liver & 6 \\
Lymph-node & 9 \\
Median weight loss & $10 \%$ \\
$<1 \%$ & 1 \\
$1-5 \%$ & 4 \\
$6-10 \%$ & 7 \\
$>10 \%$ & 11 \\
\hline
\end{tabular}

a life expectancy of 3 months or more; age 75 years or less; no prior chemotherapy; no symptomatic brain metastases; adequate bone marrow and kidney function (clearance $>60 \mathrm{ml} / \mathrm{min}$ ); measurable disease-if the primary tumour was the only marker lesion and not previously irradiated, the disease was considered evaluable and monitored by barium radiogram, computed tomography and endoscopy with biopsies; and a guaranteed food intake (in cases of severe stenosis, a prosthetic intubation was performed). Patients with a probability of $<0.05$ of not developing severe CNS toxicity after ifosfamide/mesna infusion, calculated by means of serum albumin and creatinine concentration according to the monogram described by Meanwell et al. [1], were not eligible.

Treatment consisted of prehydration with $500 \mathrm{ml}$ saline $0.9 \%$ for $2 \mathrm{~h}$ followed by $200 \mathrm{ml}$ mannitol $20 \%$ over $30 \mathrm{~min}$ and mesna $1 \mathrm{~g} / \mathrm{m}^{2}$ intravenously. Ifosfamide was given as a $48 \mathrm{~h}$ infusion in a total dose of $6 \mathrm{~g} / \mathrm{m}^{2}$ together with $6000 \mathrm{ml}$ dextrose/saline and mesna $3 \mathrm{~g} / \mathrm{m}^{2}$. Posthydration was given with $2500 \mathrm{ml} \mathrm{dex-}$ trose/saline over $16 \mathrm{~h}$ together with mesna $2 \mathrm{~g} / \mathrm{m}^{2}$. Courses were repeated every 4 weeks. Patients were evaluable for response after two courses, and evaluable for toxicity after one course. In cases of clear progression after the first course, the response was evaluated as early progression. In cases of stable disease after any two cycles, treatment was stopped. The maximal duration of treatment was six cycles or until progression of disease or intolerable toxicity, physical or mental. The recommended guidelines for the criteria of evaluation and toxic effects proposed by the WHO (1979) [2] were followed. Blood counts were performed weekly.

\section{RESULTS}

Of 25 entered patients, 1 was not eligible (adenocarcinoma of the gastric cardia without involvement of the oesophagealgastric junction area) and 1 was not evaluable for response

Correspondence to T.C. Kok.

T.C. Kok, A. van der Gaast and T.A.W. Splinter are at the Department of Medical Oncology - D 329 and H.W. Tilanus is at the Department of Surgery, University Hospital Rotterdam, Dr Molewaterplein 40, 3015 GD Rotterdam, The INetherlands.

Received 21 Feb. 1991; accepted 14 May 1991.

Table 2. Tumour characteristics $(n=23)$

\begin{tabular}{lr} 
Adenocarcinoma in Barrett's epithelium & 4 \\
Signet-ring cell carcinoma in Barrett's epithelium & 2 \\
Grade of malignancy (ICD-O) & \\
Grade 2 & 15 \\
Grade 3 & 7 \\
Unknown & 1 \\
Location of primary tumour & \\
Lower oesophagus with junction area & 9 \\
Lower oesophagus with junction area and cardia & 10 \\
Junction area plus cardia & 2 \\
Unknown & 2 \\
\hline
\end{tabular}

(no response evaluation after the second course). The main characteristics of 23 cvaluable patients are shown in Table 1; tumour characteristics are shown in Table 2. Most patients were men with a fair performance status, notwithstanding a significant weight loss, who had metastatic cancer at first presentation.

Toxicity data of 24 patients are shown in Table 3, graded according to WHO criteria (1979). Of a total of 63 courses, the median number was only two. There was no treatment delay because of cytopenia; only once did a leucocyte nadir of 1.1 in combination with fever require a dose reduction of $25 \%$ for the next courses. In 2 cases a serious infection (WHO grade 3) required hospital admission for intravenous antibiotic therapy (WBC nadir 1.7 and $1.1 \times 10^{9} / 1$ ). No serious renal toxicity nor any CNS toxicity were seen during the study. Reasons for going off study were: serious subjective toxicity $(n=1)$, progressive disease during treatment (11), stable disease after two courses (7) and end of protocol treatment (4). 1 patient experienced rapid deterioration of his condition after the first course: no response evaluation was done.

Among 23 patients evaluable for response, 1 achieved a complete response (CR: response duration $29+$ months) and 1 a partial response (PR: response duration 7 months).

The patient who achieved a CR was a 47-year-old man, with a

Table 3. Toxicity profile $(n=24)$

Total courses

Median courses

Dose reduction

Treatment delay (cytepenia)

Median WBC nadir

Median platelet nadir

Toxic death

\begin{tabular}{lrrrrr} 
& \multicolumn{5}{c}{ WHO grades } \\
\cline { 2 - 7 } & 0 & 1 & II & III & IV \\
\cline { 2 - 7 } & 13 & 2 & 6 & 3 & \\
WBC & 22 & & & 2 & \\
Infection & 24 & & & & \\
Platelets & 1 & 6 & 13 & 4 & \\
Nausea, vomiting & 24 & 3 & 15 & 6 & \\
Hair & 23 & 1 & & & \\
Neurotoxicity & 23 & 1 & & & \\
Renal & & & & & \\
Cutaneous & & &
\end{tabular}

$4.0(1.1-6.3) \times 10^{9} / 1$

$262(174-571) \times 10^{9} / 1$

0

$$
\begin{gathered}
2(1-6) \\
1 \times \\
0 \times
\end{gathered}
$$


poorly differentiated adenocarcinoma in Barrett's epithelium and microscopically proven metastatic lymph-nodes in the coeliac region at laparotomy. He refused surgical treatment after six courses of chemotherapy, and is now, $2 \frac{1}{2}$ years later, in perfect condition without clinically detectable tumour (endoscopy plus biopsies). The median survival time of all evaluable paticnts was 7 months (range 2-54) after start of treatment and 3.5 months after stopping chemotherapy. 3 patients are still alive, with a follow-up of 2, 3 and $24+$ months, respectively, after stopping treatment.

\section{DISCUSSION}

Although 2 patients in this phase II study achieved a well documented major regression, ifosfamide seems to have minor activity in untreated patients with advanced adenocarcinoma of the oesophagus or oesophageal-gastric junction area. Such a lack of response has also been documented for epidermoid carcinoma of the oesophagus in two other trials $[3,4]$. However, we could not confirm the severe toxicity, especially myelosuppression, described in these reports. Several factors could play a role in this discrepancy. For example, our patients had a better performance status than those described by Ansell et al. [3]. More than half of the patients in Nanus et al.'s report were pretreated with radiotherapy and/or chemotherapy [4]. Concern- ing the dose and schedule of ifosfamide, we administered $6 \mathrm{~g} / \mathrm{m}^{2}$ as a continuous infusion over $48 \mathrm{~h}$ instead of $7.5 \mathrm{~g} / \mathrm{m}^{2}$ over 5 days as daily short intravenous infusions. On the other hand, our data on bone marrow suppression are not different from those of Ansell et al., and clearly less serious than those of Nanus et al., who experienced 18 episodes of WBC count nadir $<1000$ in 59 cycles of therapy against 0 in our series of 63 cycles.

In conclusion, ifosfamide, given in a dose of $6 \mathrm{~g} / \mathrm{m}^{2}$ over $48 \mathrm{~h}$, has a low activity as first-line treatment in patients with adenocarcinoma of the oesophagus. The application of a continuous administration over $48 \mathrm{~h}$ may result in a more favourable toxicity profile than observed in fractionated regimens using daily short intravenous infusions for several days.

1. Meanwell CA, Blake A, Kelly KA, Honigsberger L, Blackledge G. Prediction of ifosfamide/mesna associated encephalopathy. Eur $\mathcal{F}$ Cancer Clin Oncol 1986, 22, 815-819.

2. WHO. Handbook for Reporting Results of Cancer Treatment. WHO Offset Publication no. 48, Geneva, WHO, 1979.

3. Ansell SM, Alberts AS, Falkson G. Ifosfamide in advanced carcinoma of the esophagus: a phase II trial with severe toxicity. Am $\mathrm{J}$ Clin Oncol 1989, 12, 205-207.

4. Nanus DM, Kelsen DP, Lipperman R, Eisenberger M. Phase II trial of ifosfamide in epidermoid carcinoma of the esophagus: unexpectant severe toxicity. Invest New Drug 1988, 6, 239-241.

\title{
Clinical Outcome of Postoperative Adjuvant Immunochemotherapy with Sizofiran for Patients with Resectable Gastric Cancer: a Randomised Controlled Study
}

\author{
Shigeru Fujimoto, Hisashi Furue, Tadashi Kimura, Tatsuhei Kondo, \\ Kunzo Orita, Tetsuo Taguchi, Koichi Yoshida and Nobuya Ogawa
}

Adjuvant immunochemotherapy using the antitumour polysaccharide sizofiran (SPG), an extract from the culture broth of Schizophyllum commune Fries, was prescribed randomly for 386 Japanese patients with resectable gastric cancer. Although the overall survival probability for 5 years did not differ between the SPG and control groups, in 264 patients with curatively resected cancer, the probability to 5 year survival and to recurrence in the sizofiran-administered patients was better than in the controls. In the multivariate analysis, four of six prognostic factors correlated with the prognosis of the 264 patients who underwent curative surgery, that is, nodal involvement $\left(\chi^{2}=21.426, P=<0.0001\right)$, age distribution $\left(\chi^{2}=9.262, P=0.010\right)$, sizofiran administration $\left(\chi^{2}=6.507, P=0.011\right)$, and primary tumour size $\left(\chi^{2}=9.345, P=0.025\right)$. Thus, patients with a curatively resected gastric cancer had a better prognosis when sizofiran was prescribed in combination with antitumour drugs.

Eur.7 Cancer, Vol. 27, No. 9, pp. 1114-1118, 1991.

\section{INTRODUCTION}

WHILE AN early diagnosis of gastric cancer is now feasible, extensive resection has to be done for those in the advanced stage of the disease. The long-term survival of these patients depends on the extent of micrometastasis present at the time of surgery, that is, the micrometastasis responsible for the recurrence has to be given due consideration. For this purpose, adjuvant cancer chemotherapy has been prescribed [1-3], but since anticancer drugs have immunosuppressive effects, adjuvant immunotherapy is required to gain an extended time of survival.

Sizofiran ( $S P(j)$, a glucan extracted from culture medium 